\title{
CONDORCET : DE L'ÉCONOMIE POLITIQUE AUX SCIENCES DE LA SOCIÉTÉ
}

\author{
N'y a-t-il pas un ancien qui a prétendu que le grand \\ Etre avait abandonné l'arrangement de cette terre à \\ un génie médiocre qui y avait fait beaucoup de sot- \\ tises? Cet ancien avait raison... \\ Lettre de Condorcet à Turgot, 11 août 1772.
}

Ce fragment de biographie intellectuelle voudrait dissiper un paradoxe. Dans l'œuvre de Condorcet, l'économie occupe une place considérable. L'auteur a participé à tous les débats de son temps avec une aisance, une clarté compréhensibles si l'on songe au faible niveau d'abstraction de la discipline. Couramment pratiquée par des amateurs éclairés, celle-ci ne reste-t-elle pas jeu d'enfant pour un académicien versé dans l'analyse mathématique et le calcul des probabilités ?

Surprise. La réputation de Condorcet économiste ne s'est jamais affirmée. Les contemporains tiennent visiblement ses écrits pour secondaires. Les grands auteurs du temps les citent peu et souvent pas du tout. Faut-il croire que cet écrivain demeure un publiciste parmi d'autres dans une génération qui en a tant compté ? L'incessante activité qu'il a dépensée dans le journalisme expliquerait ce jugement. Or les historiens du XIXe et du XXe siècle ne l'ont pas démenti. On a scruté le rôle académique du savant, ses travaux mathématiques, la théorie du suffrage, les projets d'enseignement, l'action politique révolutionnaire ${ }^{1}$. Les textes économiques n'ont suscité qu'un médiocre ouvrage.

1. Pour s'en tenir à quelques travaux importants : Léon CAHEN, Condorcet et la Révolution française, Paris, Alcan, 1904 ; J. Salwyn SCHAPIRO, Condorcet and the Rise of Liberalism, New York, Harcourt, Brace \& Co, 1934 ; Gilles-Gaston Granger, La Mathématique sociale du marquis de Condorcet, Paris, P.U.F., 1956 ; Roshdi RASHED, ed., Condorcet. Mathématique et société, Paris, Hermann, 1974 ; Keith Michael BaKER, Condorcet. From Natural Philosophy to Social Mathematics, Chicago, University of Chicago Press, 1975 ; Catherine KINTZLER, Condorcet, l'instruction publique et la naissance du citoyen, Paris, Le Sycomore, 1984 ; Michèle CRAMPE-CASNABET, Condorcet, lecteur des Lumières, Paris, P.U.F., 1985. 
Sans doute ce désintérêt forme, à lui seul, une faible présomption. Mais il existe d'autres critères et ils suggèrent le même constat. J. A. Schumpeter publie par exemple en 1958 une magistrale History of Economic Analysis ${ }^{2}$, qui constitue la base du savoir contemporain. Avec neuf références, certes Condorcet n'est pas oublié, mais cette place reste bien modeste en regard des principaux auteurs du XVIIIe siècle : Quesnay 58 citations, Turgot 46, A. Smith 149. Elle n'autorise guère la révision du dossier. Et pourtant, il faut en venir là.

Condorcet fût-il un médiocre économiste, une telle faiblesse mériterait commentaire puisqu'elle se constate chez un homme qui fonde la mathématique sociale et propose l'analyse des choix collectifs. Ou bien est-ce le contraire ? Alors il faut savoir pourquoi le mathématicien déconcerte ses contemporains et se prête si mal ultérieurement à l'insertion dans les problématiques de la discipline. Toutes les hypothèses valent donc examen.

\section{L'ÉCONOMIE DE CONDORCET}

La lecture des œuvres écarte un doute initial. La culture économique du mathématicien était bien l'une des plus complètes du siècle ${ }^{3}$. Ses propres références, souvent accompagnées de réflexions critiques, surprennent par leur densité. Elles annoncent d'ailleurs une rupture avec les mœurs intellectuelles d'une époque où l'on se contentait d'allusions incertaines et de plagiats tacites. Elles permettent de reconstituer un horizon culturel.

Celui-ci présente une parfaite conformité avec la réinterprétation de la discipline que les physiocrates ont proposée. Parcourir les citations de Condorcet, c'est en fin de compte réécrire la « Notice... des différents écrits modernes qui ont concouru en France à former la science de l'économie politique " que Dupont de Nemours publiait dans les

2. Eugène Caillaud, Les Idées économiques de Condorcet, Poitiers, Bousrez, 1908 ; Joseph Aloys SCHUMPETER a été traduit tardivement en français : Histoire de l'analyse économique, Paris, Gallimard, 1983, 3 vols.

3. On utilise ci-dessous l'édition classique: Euvres de Condorcet publiées par A. Condorcet $O^{\prime}$ Connor et M.F. Arago, Paris, 1847-1849, 12 vols, avec la référence abrégée : $E$ Euvres. Cette précieuse publication exclut les travaux mathématiques ; elle n'est pas exempte de défauts et de flou dans les datations. Pour deux textes fondamentaux, on s'est reporté aux éditions originales: Journal d'instruction sociale, par les citoyens Condorcet, Siéyès et Duhamel, Paris, 1793 (six numéros) ; Esquisse d'un tableau historique des progrès de l'esprit humain, Paris, an III (1795). Un travail plus étendu exigerait le retour aux manuscrits conservés à la bibliothèque de l'Institut et à la Bibliothèque nationale. 
Éphémérides en $1769^{4}$. Dans les deux cas, une même séquence d'auteurs apparaît. La préhistoire de la science s'étend de Boisguilbert à Cantillon et comprend l'analyse critique de Melon et Dutot. Puis les questions protocolaires s'établissent avec les disciples de Vincent de Gournay : l'abbé Morellet et Turgot, tandis que les principes se fixent dans les textes de François Quesnay. Condorcet a pratiqué en effet les grands physiocrates : le marquis de Mirabeau, Dupont, l'abbé Roubaud; il a dépouillé leur périodique et les agronomes proches de l'école, Trudaine ou Duhamel du Monceau ; il connaît les contradicteurs de Quesnay : Linguet, Galiani ; il a lu la Théorie du luxe de Butel-Dumont (1771). On relève juste deux lacunes dans ce panorama classique, Graslin et Forbonnais.

Condorcet ne s'est pas limité aux travaux des auteurs déjà consacrés. $\mathrm{Au}$ fil des ans, ses références économiques s'enrichissent d'œuvres majeures récentes : le grand livre de Necker, De l'administration des finances de la France (1784), la fiscalité grâce à Moreau de Beaumont, la spéculation sapitaliste avec le comte de Mirabeaus. Les textes que l'académicien semble ignorer sont également ceux que le XVIII siècle, à tort ou raison, plaçait èn seconde ligne, comme le Traité de la circulation et du crédit (I. de Pinto en 1771), le Traité des richesses (A.-N. Isnard, 1781), De l'économie politique moderne (J. F. de Herrenschwand, 1786).

Mais il lit mieux que bien des contemporains les œuvres étrangères. $S$ 'il ignore, comme tout le monde en France, la statistique descriptive des caméralistes allemands, il possède Pietro Verri (Meditazioni sull' Economia politica, 1771) ; il connaît admirablement les grands auteurs anglais : J. Steuart, An Inquiry into the Principles of Political Economy (1767, traduction française en 1789), les débats financiers au parlement de Londres, les interventions de lord Stanhope et de Fox. Enfin, comme tant d'autres en France, Condorcet a rêvé d'une édition critique de la Richesse des nations que la Révolution ne lui laisse pas le loisir d'achever' .

4. Éphémérides du citoyen ou Bibliothèque raisonnée des sciences morales et politiques, année 1769, sur huit numéros à partir de janvier.

5. J. L. MOREAU DE BEAUMONT, Mémoires concemant les droits et impositions en Europe..., Paris, 1787-1789, 5 vols ; Honoré Gabriel, comte de MirabeaU, Dénonciation de l'agiotage au Roi..., s. 1., 1787.

6. La réception d'Adam SMITH a été foudroyante de ce côté de la Manche. L'édition originale, An Inquiry into the Nature and Causes of the Wealth of Nations, Londres, 1776, est connue du Journal des savants dès février 1777, ce périodique en publie des extraits. La première traduction française complète date de 1778-1779 ; rééditions et nouvelles traductions s'accumulent en 1781, 1786, 1788, 1789. Condorcet ouvre les colonnes de la Bibliothèque de l'homme public à un long compte rendu de Smith en 1790 (t. II et III). La même année, le libraire Buisson annonce à Paris une nouvelle édition des Recherches, 
Sa culture s'étend aussi aux domaines limitrophes. L'analyse économique du $\mathrm{XVIII}^{\mathrm{e}}$ siècle tarde en effet à conquérir ses champs autonomes. Elle entretient des liens toujours privilégiés avec l'Arithmétique politique. Condorcet lit les fondateurs anglais W. Petty, Graunt, King, Davenant et retient leur statistique purement descriptive. Il suit l'orientation de plus en plus démographique de cette discipline, sa rencontre avec les probabilités qui autorise les calculs d'espérance de vie et la fixation des rentes viagères. Ses références couvrent ainsi les deux versants : la collecte empirique des données (Deparcieux, Wargentin, Buffon) et l'outil mathématique (Fermat, de Moivre, Montmort, Euler, les Bernoulli, Bayes, Price, Laplace) ; il est lui-même orfèvre en la matière.

Enfin, l'économie politique de son temps demeure insérée dans les "sciences morales et politiques" au sein desquelles elle entend jouer le rôle principal. Cette expression qui caractérise un bloc de connaissances en instance de coupures apparaît d'ailleurs chez un contemporain, l'abbé Baudeau, pour la première fois dans les années 1760 . Nulle défaillance, là non plus, dans la culture de Condorcet dont les strates reflètent les étapes européennes de la pensée politique : les fondateurs, Machiavel et Bodin, les philosophes du droit naturel, Pufendorf, les adversaires de l'absolutisme royal, Fénelon, l'abbé de SaintPierre, les théoriciens anglais de Locke à Hume, tous les écrivains des Lumières, Montesquieu, Helvétius et Voltaire, Rousseau et Mably, d'Holbach ou l'abbé Raynal.

À ces connaissances livresques bien choisies, Condorcet ajoute le privilège des liens académiques et l'entrée dans les salons où l'on parle d'économie. Il est lié avec la duchesse d'Enville qui recevait d'Alembert, Turgot, Quesnay, Mirabeau, Dupont de Nemours, Baudeau ; il est l'ami de La Rochefoucauld-Liancourt, de Madame du Deffand et le fidèle habitué du salon de Julie de Lespinasse où se regroupent depuis 1769 les Encyclopédistes et toutes sortes d'économistes : Morellet, Turgot, Chastellux, Galiani, lord Shelburne ; il côtoie, chez Madame Helvétius, d'Holbach, l'abbé Raynal, Condillac, Destutt de Tracy. Il appartient, comme Lavoisier, à la Société libre d'èmulation, fondée par l'abbé Baudeau en décembre $1776^{7}$. Les

traduite sur la quatrième anglaise et suivie " d'un volume de notes par M. le marquis de Condorcet ". La publication s'achève en 1791 sans la participation de l'académicien. Celle-ci est encore annoncée en vain dans l'édition suivante de 1791-1792 (à Avignon, chez J. J. Niel). Ces deux éditions sont décrites dans le Catalogue de la Kress Library sous les cotes B 1986 et B 2210.

7. Sur les salons proches des physiocrates, beaucoup de renseignements dans Georges WeUlersse, Le Mouvement physiocratique en France de 1756 à 1770, Paris, Alcan, 1910, 2 
Éloges qu'il doit écrire pour l'Académie des sciences à la disparition de Trudaine, de Mignot de Montigny, Duhamel du Monceau, Euler, Wargentin le ramènent par la voie biographique aux frontières des sciences sociales. Son activité mathématique, de l'analyse à l'application des probabilités, manifeste un parcours analogue. C'est donc la trajectoire de l'académicien dans la théorie économique qu'il faut saisir maintenant qu'on s'est assuré de ses connaissances.

L'intérêt de Condorcet apparaît pour la première fois dans sa correspondance avec Pietro Verri en novembre 1771, à la publication des Meditazion $i^{8}$. Un an plus tôt, l'académicien avait lié connaissance avec Turgot. Il doit à cette rencontre son initiation aux matières de gouvernement. En 1774, l'intendant de Limoges devient contrôleur général des Finances. Comme plusieurs des habitués du salon Lespinasse, Condorcet s'engage avec ferveur aux côtés du ministre qui lui procure l'inspection des Monnaies ${ }^{9}$. Il prépare les dossiers fiscaux du contrôleur : l'abolition de la corvée, la réforme de la Ferme générale ; il réfléchit à l'enquête sur les poids et mesures, aux projets de voies navigables. Il accompagne l'action ministérielle, de ses premiers plaidoyers en faveur de la liberté économique ${ }^{10}$.

La prompte disgrâce de Turgot, le 12 mai 1776 , a provoqué une déchirure dans la vie de Condorcet. Certes l'affection y avait sa part : « Un grand homme, dit-il, que j'ai tendrement chéri, dont l'amitié m'a été si douce et si utile... un de ces sentiments délicieux et tristes qui

vols; ID., La Physiocratie à la fin du règne de Louis XV (1770-1774), Paris, P.U.F., 1959 ; ID., La Physiocratie sous les ministères de Turgot et de Necker (1774-1781), Paris, P.U.F., 1950 ; ID., La Physiocratie à l'aube de la Révolution (1781-1792), éd. par Corinne Beut. LER, Paris, Éd. de l'E.H.E.S.S., 1985. Les attaches de Condorcet avec les salons du xvme siècle sont évoquées par L. CAHEN, op. cit. supra n. 1, p. 23-24, K. M. BAKER, op. cit. supra n. 1, p. 16-23. Voir aussi, Jean-Dominique DE LA RoChefoucauld, Claudine Wolikov, Guy IKNI, Le Duc de La Rochefoucauld-Liancourt, Paris, Perrin, 1980. La rivalité de Madame du Deffand et de Julie de Lespinasse a scindé les fidèles des deux salons, cf. Benedetta Craveri, Madame du Deffand et son monde, Paris, Seuil, 1987 ; ce livre a le mérite de recopier beaucoup de citations.

8. L'ouvrage de Pietro VERRI est traduit rapidement en français par Ch. Mingard, Réflexions sur l'économie politique, Lausanne, 1773. La lettre de Condorcet au comte Verri le 7 novembre 1771 se trouve in CEuvres, t. I, p. 283-284. Sur les contacts des frères Verri avec la France, cf. Viaggio a Parigi e Londra (1766-1767), Carteggio di Pietro e Alessandro Verri, testo, prefazione, note e indici a cura di Gianmarco Gaspari, Milan, Adelphi Ed., 1980.

9. Dans sa Vie de Turgot, in Euvres, t. V, p. 105 sq., Condorcet évoque avec nostalgie le ministère de 1774-1776: "Tous les hommes qui cultivaient les sciences, les lettres, les arts... étaient traités avec distinction. On était sûr d'être écouté, d'être accueilli pourvu qu'on eût à lui dire quelque chose qui pût contribuer au bonheur public. »

10. Ce sont les petites brochures de 1774-1775: Lettres sur le commerce des grains, Lettre d'un laboureur de Picardie, Réflexions sur les corvées, "Monopole et Monopoleur " dans les Suppléments de l'Encyclopédie et l'ouvrage de 1776, Réflexions sur le commerce des blés. 
deviennent une partie de notre existence et ont le pouvoir de nous la rendre plus chère. " Mais le renvoi du ministre n'exige pas seulement le deuil d'un pouvoir politique et mondain, il sanctionne un échec intellectuel. Le 5 juin 1776, l'académicien confie à Voltaire :

« Cet événement a changé pour moi toute la nature [...] Adieu, mon cher et illustre maître; nous avons fait un beau rêve, mais il a été trop court. Je vais me remettre à la Géométrie et à la Philosophie. Il est bien froid de ne travailler que pour la gloriole quand on s'est flatté pendant quelque temps de travailler pour le bien public " ".

Le programme interrompu laissait à l'abandon les assemblées municipales et provinciales, la réforme des finances publiques (la liquidation de la dette, la gestion du domaine royal, la fiscalité, l'extinction progressive des droits seigneuriaux et ecclésiastiques), la politique des transports. Toutes questions qui vont constituer des enjeux nationaux dans la période révolutionnaire et le champ de réflexion privilégié de Condorcet de 1787 à $1793^{12}$, après dix ans de silence.

La gémellité intellectuelle des deux hommes traverse en effet les événements, comme le démontre le parallélisme des biographies. Au lendemain de 1776, l'ancien contrôleur général des Finances n'écrira plus une ligne d'économie. Il tourne ses regards vers l'hydraulique, la physique et l'astronomie. Il médite un ouvrage sur la philosophie du savoir que la mort interrompt le 18 mars 1781 . En un mot, le politique est devenu le savant. Cette allusion aux concepts de la pensée wébérienne se justifie si l'on songe que Condorcet a tenté toute sa vie de parcourir le même chemin en sens inverse. Cette fascination mutuelle de chacun pour son double éclaire un projet de pensée commun, bien au-delà de l'anecdote biographique. Le dernier livre de Turgot devait constituer l'aboutissement d'un discours-programme de jeunesse : celui qu'il prononça, étudiant à la Sorbonne en décembre 1750 , le "Tableau philosophique des progrès successifs de l'esprit humain ${ }^{13}$; ce travail inachevé, n'est-ce pas celui que Condorcet, luimême menacé de mort, écrit en 1793-1794, l'Esquisse d'un tableau historique des progrès de l'esprit humain?

11. Nombreux témoignages sur la collaboration des deux hommes dans la Vie de Turgot, in CEuvres, t. V ; la citation de ce paragraphe est extraite de l'Avertissement. La lettre à Voltaire figure au tome I des Euvres, p. 115.

12. Condorcet s'est attardé sur le programme du contrôleur général dans la Vie de Turgot, in Euvres, t. V, p. 113-149. Le livre de l'académicien serait à comparer avec celui de Pierre Samuel Dupont de Nemours, Mémoire sur la vie et les ouvrages de M. Turgot ministre d'État, Philadelphie, 1782.

13. Gustave SCHELle, Euvres de Turgot et documents le concernant, Paris, Alcan, 1913-1923, 5 vols, t. I, p. 214-235. 
Du reste, dès 1776 l'attitude du mathématicien fut identique à celle de Turgot. On mesure la profondeur de son désarroi dans l'étude historique qu'il consacre en 1776-1777 au chancelier Michel de l'Hospital ${ }^{14}$. En esquissant le portrait d'un ministre méconnu des souverains, incompris des peuples, en évoquant l'échec de cet esprit tolérant, réformateur, l'écrivain propose à ses contemporains un exercice d'herméneutique, la méditation de leur histoire nationale la plus proche. Puis, de ce premier mémorial au second, la Vie de Turgot en 1786, Condorcet adopte comme le Contrôleur général la même réserve en matière économique. On relève tout juste de furtives analyses dans le Mémoire sur le canal de Picardie (1780), le Rapport [...] pour la réformation du cadastre de la Haute-Guyenne (1780-1781), dans les Réflexions sur l'esclavage des nègres (1781). Il faut, d'ailleurs, noter la concordance de ce vide avec l'affaissement temporaire des publications françaises. Le retour à l'économie s'amplifiera après 1785 ; Condorcet participe à l'intermittence de ces flux ${ }^{15}$.

En 1786, dans la Vie de Turgot, l'académicien paraît de prime abord écrire un deuxième ouvrage historique ; il dresse le bilan des actes et des thèses économiques du ministre défunt. Cependant, il faut bien convenir que cette œuvre est susceptible d'une autre lecture. Comparons-la à la biographie parallèle publiée par Dupont de Nemours ; la différence est extrême. Pour Condorcet, le ministre n'est plus que le truchement qui lui permet d'exposer sa propre vision économique du royaume. Cette vie qui s'est achevée en 1781 parle plus éloquemment du malaise des années 1786-1789 et des tâches scientifiques à assumer ultérieurement que de 1775.

Dès lors, deux traits significatifs sont à retenir dans cet ouvrage. Condorcet a construit un Turgot physiocrate. L'intéressé, mais Dupont de Nemours aussi bien, auraient fait d'importantes réserves, pleinement ratifiées aujourd'hui ${ }^{16}$. Le ministre est en outre crédité

14. Éloge de Michel de l'Hospital, chancelier de France, in CEuvres, t. III, p. 463-566. Ce texte est la version développée d'un discours prononcé à l'Académie française en 1777. Rappelons que l'orateur n'est devenu membre de cette compagnie qu'en 1782. L'évocation historique nous reporte au xvle siècle : le chancelier a vécu de 1507 à 1573 .

15. Publications économiques françaises : $1770-1774=247$ titres; $1775-1779=267$; $1780-1784=172 ; 1785-1789=657$. Pour un bref aperçu statistique des éditions, cf. Jean-Claude PERrot, "Les publications françaises d'économie politique (xVIle-xville siècles) ", Bulletin de la Société d'histoire moderne, 25, 1985, p. 21-26.

16. Turgot a constamment diffusé les cuuvres des physiocrates, tout en marquant ses différences. Dupont de Nemours avait corrigé de son propre chef le manuscrit du ministre "Réflexions sur la formation et la distribution des richesses" pour l'imprimer dans les Éphémérides du citoyen (1770), au vif mécontentement de l'intéressé. L'histoire a définitivement souligné l'écart analytique qui sépare Turgot de Quesnay. Cf. le séminaire de Limoges pour le bicentenaire de la mort de Turgot, Christian Bordes, Jean MORAnGe, 
d'un programme de recherches que ses écrits, ou sa correspondance avec Condorcet, sont loin de dessiner avec une comparable fermeté ${ }^{17}$. Il s'agit de réinsérer l'économie dans une anthropologie et une science sociale unifiées par l'hypothèse de la perfectibilité de la raison et l'emploi d'une seule méthode : l'analyse. Le terme se charge ici de toutes les connotations qu'impliquent les mathématiques du XviII siècle. Mais Turgot n'était pas géomètre. En redessinant un ministre à sa mesure, Condorcet se forge un héritage intellectuel au moment où la crise de l'Ancien Régime restaure à ses yeux l'utilité des engagements civiques. C'est son bien propre.

De 1786 aux États Généraux, il établit les bases institutionnelles qui peuvent rénover l'économie. C'est d'abord, en 1786-1788, l'examen de l'expérience américaine, puis en 1788-1789 sa possible transposition en France ${ }^{18}$. À partir de la Révolution, Condorcet commente les débats législatifs, comme naguère les réformes de Turgot. Trois orientations principales sont à retenir : le budget de l'État en 1789-1790, la monnaie en 1790-1791 et la fiscalité de 1790 à $1793^{19}$. Deux textes testamentaires enfin s'inscrivent, durant ces années bousculées, dans le projet purement épistémologique de la Vie de Turgot. Encore sont-ils nés de l'urgence politique qui va condamner l'écrivain au silence. De là le statut d'ébauche, de panorama que souligne chacun des titres, le Tableau général de la Science qui a pour objet l'application du calcul aux

Turgot, économiste et administrateur, Paris/Limoges, P.U.F./Publications de la Faculté de droit et de sciences économiques, 1982. L'ouvrage de Claude Morilhat, La Prise de conscience du capitalisme. Économie et philosophie chez Turgot, Paris, Méridiens-Klincksieck, 1988, est paru après la rédaction de cet article. Le lecteur y trouvera de nombreux éléments qui permettent de poursuivre le parallèle Turgot-Condorcet et de percevoir ce qui les oppose tous les deux à la pure physiocratie.

17. Charles Henry, éd., Correspondance inédite de Condorcet et de Turgot, 1770-1779, publiee avec des notes et une introduction d'après les autographes de la collection Minoret et les manuscrits de l'Institut, Paris, Charavay Frères, 1883.

18. Période 1786-1788 : De l'influence de la Révolution d'Amérique sur l'Europe; in CEuvres, t. VIII ; Lettres d'un bourgeois de New Haven, in ibid., t. IX ; Lettres d'un citoyen des États-Unis, in ibid., t. IX. Période 1788-1789, Essai sur la constitution et les fonctions des assemblées provinciales, in ibid., t. VIII, et quantités de notes à l'édition de Voltaire, in ibid., t. IV.

19. Trois textes sur le budget: Plan d'un emprunt public avec des hypothèques spéciales, 1789 ; Sur les opérations nécessaires pour rétablir les finances, 1790 ; Sur la constitution du pouvoir chargé d'administrer le trésor national, 1790. Ces trois textes se trouvent dans les CEuvres, t. XI. Huit textes sur les monnaies : les cinq Mémoires sur les monnaies de décembre 1790, Sur la proposition d'acquitter la dette exigible en assignats, 1790, Des causes de la disette du numéraire..., 1790, in CEuvres, t. XI ; Mémoire sur les effets... de la nouvelle monnaie de cuivre, 1791, in Euvres, t. XII. Quatre textes sur la fiscalité : les deux mémoires Sur la fixation de l'impôt, de 1790, Sur l'impôt personnel, 1790, in Euvres, t. XI, "Sur l'impôt progressif », dans le Journal d'instruction sociale du ler juin 1793, p. 11-24. 
sciences politiques et morales, l'Esquisse d'un tableau historique des progrès de l'esprit humain ${ }^{20}$.

La fresque de cette œuvre économique peut suggérer des conclusions prématurées. Doit-on interpréter les publications de Condorcet comme des textes de circonstance sans dimension théorique ? $\mathrm{Ce}$ point de vue est peut-être exact, mais il n'est pas encore temps de l'adopter. De grands livres d'économie sont toujours sortis de moments historiques singuliers : le Détail de la France de Boisguilbert à l'effondrement du colbertisme, Money and Trade de John Law pendant les tâtonnements financiers de la banque de Londres.

On refusera également de se laisser abuser par le ton normatif de l'auteur. En réalité, les points de vue analytique et prescriptif sont consubstantiels à l'économie du XVIII siècle, précisément parce qu'elle est politique. Quesnay déduit constamment une norme des lois scientifiques qu'il estime établies. On en dirait presque autant de Malthus dans ses Principles of Political Economy de 1820.

Reste une incertitude plus générale : où placer l'œuvre de Condorcet dans une littérature qui s'étage des brochures de vulgarisation jusqu'au travail scientifique ? Avant 1789, des essayistes toujours plus nombreux se trouvent portés par la demande d'une opinion publique élargie ${ }^{21}$. Les philosophes eux-mêmes ont accordé ses lettres de noblesse à ce publicisme animé d'une éthique de la conviction. On le reconnaît à son trait polémique, à son éclectisme parfois contradictoire, à ses fluctuations doctrinales. Un exemple ? Ce serait Voltaire (Le Mondain, L'Homme aux quarante écus, les Questions sur l'Encyclopédie, la correspondance) tour à tour hostile et favorable aux derniers mercantilistes comme aux physiocrates, plume analytique légère, mais redoutable faiseur de renommée.

Il n'est pas malaisé de reconnaître à Condorcet les vertus qui font le bon bretteur, le talent de persiflage dont l'usage, en un siècle, est passé de la théologie (les Provinciales) à l'économie (les Dialogues sur le commerce des bleds de Galiani) ${ }^{22}$. La fluctuation des références théoriques apportera apparemment une présomption supplémentaire de légèreté scientifique : il est certain que Condorcet a mené des analyses économiques incompatibles entre elles de 1775 à 1793 .

20. Le "Tableau général..." " est publié en deux livraisons, la première anonyme, la seconde signée, du Journal d'instruction sociale, 29 juin 1793, p. 105-128 et 6 juillet 1793, p. 166-184. L'Esquisse, écrite đe juillet 1793 à mars 1794 dans la clandestinité, est publiée en l'an III (1795).

21. Sur ce mouvement culturel, on peut relire Jürgen Hagermas, Strukturwandel der Offentlichkeit, Hermann Luchterhand Verlag, 1962, trad. franç. L'Espace public, Paris, Payot, 1978.

22. Un exemple : la Lettre d'un laboureur de Picardie, 1775, in CEuvres, t. XI, p. 3-34. 
Pendant le ministère de Turgot, le mathématicien admet sans détour les conclusions des physiocrates. Sa définition du produit brut, des avances, du produit net, de la formation des prix est conforme aux articles de Quesnay dans l'Encyclopédie (" Grains", " Fermiers ») comme à Dupont de Nemours (De l'exportation et de limportation des grains, 1764) ; l'apologie de la liberté du commerce, la critique des monopoles et de la fiscalité indirecte, analogues à celles de Mirabeau dans sa Théorie de l'impôt (1760) ${ }^{23}$. À plusieurs reprises d'ailleurs, Condorcet revendique cette filiation.

La physiocratie de l'auteur s'est, en outre, prolongée bien au-delà des années 1774-1776. Dans la Vie de Turgot, il ne se contente pas de placer les travaux économiques du contrôleur général, ses Réflexions sur la formation et la distribution des richesses, à la source du livre d'Adam Smith ; c'est à la mesure de Quesnay qu'il apprécie l'apport de l'Inquiry. Et ses réserves sont alors de deux ordres. L'épistémologie : selon lui A. Smith compterait trop peu sur « la force irrésistible de la raison et de la vérité "; il est clair que cette critique s'appuie sur l'article "Evidence " que François Quesnay a donné en janvier 1756 dans l'Encyclopédie. L'analyse économique ensuite. Condorcet déplore à travers la Richesse des nations, l'abandon de la productivité exclusive de l'agriculture, des théories de l'impôt foncier unique et de l'investissement dans l'instruction publique ${ }^{24}$. Toutes ces thématiques viennent des physiocrates.

De 1786 à 1789, la fidélité aux économistes français persiste dans les travaux de l'académicien : De l'influence de la Révolution d'Amérique, les Lettres d'un bourgeois de Newhaven..., les Lettres d'un citoyen des États-Unis, l'Essai sur la Constitution... des Assemblées provinciales, les notes de l'Édition de Voltaire ${ }^{25}$. D'ailleurs, une telle fascination n'est pas propre à Condorcet. On observe alors dans le royaume, un été de la Saint-Martin physiocrate que renforce l'échec politique du

23. Deux témoignages de l'année 1775 : le premier dans les Réflexions sur le commerce des blés, in Euvres, t. XI, p. 196: "ce qu'il peut y avoir d'utile et de bon dans ces réflexions ne m'appartient pas [...] je n'ai point exposé comme douteuses des choses dont je ne doute pas. " Deuxième preuve dans la correspondance de l'auteur avec Turgot, in Ch. HENRY, éd., op. cit. supra n. 17, p. 229 : « Je vais tâcher d’avoir Quesnay à louer. Je suis presque sûr de m'en tirer à la satisfaction des économistes sans être obligé de mentir. "

24. CONDORCET, Vie de Turgot, in Euvres, t. V, p. 45 : critique d'A. Smith " sur ce qu'il appelle le système agricultural ", sur ses analyses fiscales. La faiblesse théorique de l'écossais tient selon Condorcet, à son refus de suivre les physiocrates : "Nous attribuons encore à la même cause l'espèce de légèreté avec laquelle il traite soit les auteurs qu'il désigne sous le nom d'Économistes français, soit la question de l'établissement d'un impôt unique, ce qui l'a entraîné dans quelques erreurs et lui a fait commettre quelques injustices. $"$

25. ID., in Euvres, textes dispersés dans les tomes IV, VIII et IX. 
premier ministère Necker et de Calonne ${ }^{26}$. Au sein même de l'Académie des sciences, Lavoisier assume jusqu'à la contradiction la pensée de Quesnay puisque, fermier général, il fortifie le système des impôts indirects. De toute la période, on retiendra moins le plaidoyer classique de Condorcet en faveur de la fiscalité foncière ou le panégyrique des propriétaires "seuls citoyens de l'État " que l'adhésion réitérée à une science économique susceptible de se formuler en propositions irrévocables : "les principes généraux de l'économie politique sont prouvés d'une manière rigoureuse, ils ne sont sujets à aucune exception réelle $"{ }^{27}$. Au sein des théories du XVIII' siècle, il n'existe qu'une vision conforme à ce postulat déterministe, c'est la thèse de l'ordre naturel de Quesnay commentée par Mirabeau et Le Mercier de La Rivière.

Cependant en 1788-1789 s'amorcent chez Condorcet, et bien souvent dans les mêmes textes, des analyses qui se détachent de la physiocratie et la contredisent ici ou là. Dans l'Essai... sur les Assemblées provinciales de 1788 , le mathématicien aborde la rémunération du travail par les deux concepts de niveau minimum naturel et de fonds des salaires ; c'est l'approche de Turgot, ce n'est plus tout à fait celle de Quesnay. Dans le même ouvrage, l'examen du machinisme et de la division du travail appartient à la pensée de Smith.

Les déplacements théoriques les plus nets concernent quatre thèmes depuis 1790. 1) L'économie sociale. Le Projet de caisse d'accumulation de Condorcet en 1790 n'est pas incompatible avec le libéralisme de Quesnay mais il lui est étranger. La lignée intellectuelle dans laquelle s'inscrit ce travail part de Viollet de Wagnon pour aboutir à Duvillard à travers Piarron de Chamousset, Faiguet de Villeneuve, de La Rocque et Lavoisier : c'est celle des calculateurs, non des économistes ${ }^{28}$. 2) L'intervention de l'État. On se reportera aux notes de l'Édition de Voltaire. Condorcet y adopte le parti de son ancien adversaire Necker

26. Sur ce point, cf. le dernier ouvrage posthume de G. Weulersse, éd. par C. BeutLER, op. cit. supra n. 7.

27. CONDORCET, De l'influence de la Révolution d'Amérique..., in CEuvres, t. VIII, p. 24.

28. J. VIOLLET DE WAGNON, L'Auteur laquais, ouvrage nouveau et fort intéressant pour la livrée, par un projet pour l'établissement d'une maison de retraite pour tous les domestiques, Avignon, 1751 ; C. H. Piarron De Chamousset, Vues d'un citoyen, Paris, 1757, 2 vols, cf. première partie «Plan d'une maison d'association "; J. FAJGUET DE VILLENEUVE, L'Économe politique, projet pour enrichir et pour perfectionner l'espèce humaine, Londres et Paris, 1763 ; A. J. DE LA ROCQUE, Établissement d'une caisse générale des épargnes du peuple..., Bruxelles, 1786 ; A. L. LAvoisıter, "Mémoires à l'Assemblée de l'Orléanais ", in Euvres publiées par les soins de S. E. le ministre de l'instruction publique et des cultes, 1864-1893, 6 vols, t. VI ; E. Du Villard de Durand, Plan d'une association de prévoyance..., Paris, 1790. Duvillard, à 19 ans, fut premier commis aux finances sous Turgot. Son plan a été examiné et approuvé par l'Académie des sciences (rapporteurs Laplace et Condorcet). Sur cette arithmétique politique, cf. François ETNER, Histoire du calcul économique en France, Paris, Economica, 1987. 
et plaide pour une politique qui atténuerait les effets de l'inégalité sociale $\left.{ }^{29} .3\right)$ Le rôle du Luxe. Dans le même texte, voici une réhabilitation des dépenses somptuaires en totale contradiction avec l'anathème des physiocrates contre le "luxe de décoration". Comme ButelDumont dont il connaît l'ouvrage, Condorcet présente l'hypothèse d'une stimulation de l'industrie par la demande, il en valorise les effets bénéfiques sur la répartition des revenus ${ }^{30}$. 4) La fiscalité. Ici la mutation théorique est parfaitement datée. Le Deuxième mémoire sur la fixation de l'impôt en 1790 reste fidèle à Mirabeau, bien que l'auteur se résigne à soutenir les finances publiques par l'emprunt, comme Turgot l'avait décidé faute de mieux en 1775. Mais, la même année, le mémoire Sur l'impôt personnel abandonne ce point de vue ${ }^{31}$. Condorcet propose un prélèvement plus que proportionnel où se combinent la taxe physiocratique sur le produit net des terres et la cote mobilière personnelle à taux variable. Mirabeau aurait jugé ce retour implicite à la Capitation d'Ancien Régime inefficace et générateur de torsion des prix, puisque les artisans la rejetteront en dernier ressort sur les consommateurs propriétaires. "Sur l'impôt progressif ", dans le Journal d'instruction sociale, achève la rupture en 1793. L'académicien abandonne le principe de la Foncière, établi en 1790 par l'Assemblée constituante, pour une ponction croissante sur toutes les formes de revenu : salaires, rentes, actions, profits.

Est-il nécessaire de montrer combien une telle proposition, au-delà du domaine proprement fiscal, ruine la pensée de François Quesnay? L'impôt de Condorcet, en s'abattant sur tous les revenus, suppose que le travail et le capital produisent également des richesses quel que soit le secteur de l'économie où ils s'appliquent. La classe artisanale n'est donc plus définie comme stérile et si son revenu dépasse sa consommation, le circuit décrit par le Tableau économique, pièce essentielle du dispositif, devient erroné. D'autre part, la progressivité de la taxe se fonde sur un calcul d'utilité marginale, celle que représentent les dépenses de l'État pour chaque particulier. Non seulement il y a là un raisonnement que Condorcet avait jadis condamné chez Daniel Bernoulli ${ }^{32}$, mais aussi le point de départ d'une autre définition de la valeur.

29. Euvres, t. IV, p. 233.

30. G. M. BuTEL-Dumont, Théorie du luxe, ou Traité dans lequel on entreprend d'établir que le luxe est un ressort non seulement utile mais indispensablement nécessaire à la prospérité des états, s.l., 1778. Le passage de Condorcet sur le sujet figure in Euvres, t. IV, p. 234.

31. Cf. ces deux textes in Euvres, t. XI, p. 436 sq. et 471 sq.

32. Daniel Bernoulul, "Specimen Theoriae novae de mensura sortis", Mémoires de l'Académie des sciences de Saint-Pétersbourg, 1738 ; Cuvres, t. II, p. 595, Éloge de Bernoulli. 
En effet, la rupture qui vient d'être soulignée ne concerne pas seulement la fiscalité. Dans le Tableau général de la science... de 1793, les mêmes formes d'éclectisme sont réunies : un énoncé de la formation des valeurs qui paraphrase Le Commerce et le Gouvernement de Condillac, un schéma de la reproduction des richesses qui combine le produit disponible de Quesnay et les vues de Turgot sur le rôle des investisseurs, une analyse des intérêts collectifs assumés par l'État, conforme à Smith. Dans le Rapport sur l'instruction publique de $1792^{33}$ et dans l'Esquisse d'un tableau historique de 1793-1794 enfin, une autre synthèse apparaît. Pour un géomètre, elle ne va pas sans paradoxe, elle proclame l'excellence de la méthode analytique, mais elle sursoit à son usage pour s'appuyer sur le récit historique et le constat empirique. En se donnant un nouvel objet d'étude : la spirale progrès du savoir, progrès technique, développement économique, croissance démographique, Condorcet est désormais aussi loin que possible de l'ordre naturel a-chronique des physiocrates. Mais il n'est pas isolé à l'Académie des sciences ; il est significatif qu'un de ses confrères, Van der Monde, ait repris en partie ce point de vue dans le cours d'économie politique de l'an III à l'École normale et que Malthus l'ait jugé assez partagé, assez faussement séduisant aussi, pour passer sa vie à le contredire ${ }^{34}$.

Les investigations précédentes suggèrent donc un bilan nuancé. Plusieurs traits, il est vrai, rapprochent Condorcet des publicistes qui forment relais entre les savoirs spécialisés et l'opinion du moment. Mais d'autres aspects de son œuvre l'en écartent absolument. L'auteur a le souci d'examiner chaque question dans le double registre de l'économie théorique et de la science appliquée. Il traite par exemple l'incidence des impôts indirects et leur suppression, une première fois en langage algébrique, une deuxième sur un exemple arithmétique ${ }^{35}$. Il agit de même en politique avec la théorie et la pratique du suffrage ${ }^{36}$.

33. Euvres, t. VII, p. 525 sq.

34. Les cours de l'École normale seront prochainement réédités avec notes et commentaires. Sur l'enseignement de Van der Monde, cf. Jacqueline HECHT, « Une héritière des Lumières, de la physiocratie et de l'Idéologie : la première chaire française d'économie politique (1795)", Cahiers de l'ISMEA, série PE, 6, 1986, p. 5-62. Malthus répond à Condorcet en composant son premier grand livre An Essay on the Principle of Population, as it Affects the Future Improvement of Society. With Remarks on the Speculations of Mr. Godwin, M. Condorcet and other Writers, Londres, 1798, trad. franç. d'Éric VıLQuIN, Paris, I.N.E.D., 1980.

35. Méthode algébrique dans la Vie de Turgot; exemple arithmétique dans l'Essai sur la constitution et les fonctions des assemblées provinciales.

36. Traitement théorique dans l'Essai sur l'application de l'analyse à la probabilité des décisions rendues à la pluralité des voix (1785), traitement pratique dans l'Essai sur la constitution... des Assemblées provinciales (1788). 
Si les deuxièmes versions sont dans chaque cas destinées à un public étendu, c'est par un souci pédagogique préalablement étayé. Ainsi les fluctuations théoriques de l'auteur ne sont pas destinées à flatter une opinion publique versatile ; elles précèdent au contraire les exposés de vulgarisation, elles méritent d'être prises au sérieux.

Et quel sens leur donner, si l'on n'y voit d'abord le reflet du changement historique? L'économie française de l'époque révolutionnaire n'est plus celle que les physiocrates ont théorisée dans les années 1750. Il était inévitable, il était légitime que la science modifie ses objets et ses méthodes pour penser l'accélération des événements, le développement de la manufacture, la complexité montante du domaine financier. Dans ce cas, il faut seulement se demander si la mathématique sociale de Condorcet rend compte en 1793 du changement dans l'économie et des mutations théoriques qui l'appréhendent. Le parcours épistémologique de l'écrivain resterait alors cohérent et il serait plus intéressant que le contenu daté de ses commentaires sur le commerce des blés ou sur l'impôt.

LA METHODE DES SCIENCES ÉCONOMIQUES ET SOCIALES

Revenons à la physiocratie. Il importe de savoir jusqu'où pouvait aller l'adhésion de l'académicien en 1775 car elle implique une démarche scientifique. Les recherches de François Quesnay commencent par des observations exemplaires d'exploitations agricoles choisies parmi les meilleures et les moins bonnes du royaume. Elles lui permettent de caractériser le stade préindustriel de l'économie ${ }^{37}$. Par l'analyse fine de leur fonctionnement, il élabore une série de notions comptables telles que les concepts de produits brut et net : produit brut $=$ avances primitives + avances annuelles + produit net ; produit net $=$ rente + dîme + impôt royat. Ces définitions sont mises en œuvre pour étudier des domaines spécifiques, la fiscalité par exemple, ou pour constituer une théorie générale : le Tableau économique de la circulation des richesses entre les classes de producteurs, de propriétaires et d'artisans. La valeur scientifique de la construction se mesure à travers deux épreuves successives : la pertinence de concepts bien analysés, la cohérence des déductions.

37. Sur cette démarche, J.-C. PERrot, « La comptabilité des entreprises agricoles dans l'économie physiocratique », Annales E.S.C., mai-juin 1978, p. 559-579. 
Cette démarche est évidemment à la source de la fascination de Condorcet. Elle se trouve parfaitement conforme à celle des mathématiques ou des sciences de la nature ; c'est la seule qui puisse produire un savoir démontré :

"En cherchant ce qui donne à la géométrie [au sens du XviII siècle] cet avantage, on voit qu'elle n'emploie aucun terme qu'elle ne l'ait défini, que jamais le sens de ce terme ne varie et qu'ainsi on peut, dans chaque proposition, en substituant à chaque terme sa définition, parvenir à des propositions évidentes par elles-mêmes et à des notions simples qu'il ne faut plus ni prouver ni définir [...] Cette méthode est applicable aux sciences mêmes de faits, parce qu'alors une propriété donnée par l'expérience, ou un fait observé y tient lieu des notions simples, des propositions évidentes par elles-mêmes, qui ne doivent plus être ni définies ni prouvées " ${ }^{38}$.

De tous les commentaires que mériteraient ces lignes, un seul importe maintenant. Les mathématiques ne se bornent pas à accumuler de l'outillage pour les sciences physiques, elles constituent la partie la mieux formée d'un savoir qui traite le réel partout de la même manière. Dès lors la réussite de la physiocratie, à supposer qu'elle soit bien avérée, tient à l'emploi de ce modèle scientifique universel, à la construction d'une langue analytique. Ce pari gagné lui assurerait une sorte d'hégémonie sur l'ensemble des sciences morales et politiques demeurées précritiques.

Condorcet n'a jamais douté que l'entreprise soit à la fois ardue et possible. Dans le même Éloge de Pascal de 1776, il ajoute :

"Si l'application de cette méthode est facile dans presque toutes les sciences naturelles, elle devient difficile dans les sciences morales, parce que la plupart des termes [...] sont employés dans l'usage ordinaire avec un sens vague et confus et qu'il faut, après en avoir fixé le sens, veiller toujours à ce qu'il n'arrive jamais de les employer dans le sens vulgaire."

Des remarques analogues, d'ailleurs partagées par beaucoup d'économistes au même moment, Condillac, Graslin, viennent encore sous la plume de Condorcet dans le prospectus du Journal d'instruction sociale $^{39}$. On se reportera à la célèbre coupure entre « la langue du

38. CEuvres, t. III, Éloge de Blaise Pascal, p. 567-634 et spécialement, p. 613-614. L'édition des Pensées, préparée par Condorcet, sort en 1776, une deuxième édition paraît en 1778 .

39. Ibid., p. 614 ; Journal d'instruction sociale, prospectus, ler juin 1793 : « Une des principales causes du peu de progrès des sciences morales et politiques [...] c'est l'imperfection de la langue qu'elles emploient "; même idée chez Condillac, Le Commerce et le Gouvernement, éd. par Gilbert-Urbain GuILlaumin, Collection des principaux économistes, Paris, Guillaumin, 1847, t. XIV, p. 247 ; J. J. L. GrASLIN, Essai analytique sur la richesse et sur l'impôt, Londres, 1767, récuse les concepts des physiocrates, mais non la nécessité de telles définitions. 
réel ": c'est-à-dire le langage scientifique, et " la langue de convention ": le proto-langage de la vie quotidienne, argumentée dans l'Esquisse ${ }^{40}$. La langue universelle qui comblerait ce fossé dans l'avenir a valeur de projet scientifique et peut-être d'eschatologie. Mais elle est d'ores et déjà en construction dans « la morale, la politique, l'économie publique " et rien n'altérera aux yeux de Condorcet le mérite de François Quesnay qui sut donner à son travail le bon départ d'une langue bien faite.

Â partir de là, on l'a dit, le raisonnement économique des physiocrates engendre, more geometrico, les " propositions évidentes " et les " notions simples" du circuit des richesses. Comment vérifier leur pertinence ? À cette question canonique, les économistes de l'école en substituent une autre qui, paraissant rapprocher leur domaine des sciences de la nature, l'en éloigne peut-être radicalement; ils se demandent de quelle réalité la théorie peut rendre compte. La réponse figure en toutes lettres dans le titre de l'ouvrage de Le Mercier de La Rivière auquel François Quesnay a collaboré en 1767. C'est « l'ordre naturel et essentiel des sociétés politiques " que les auteurs veulent identifier. Dans ce titre-programme, l'adjectif "essentiel" a deux acceptions ; il se réfère, par-delà les phénomènes, à leur être caché, à l'ontologie sociale ; ainsi la science passe subrepticement à la métaphysique, ou du moins à la philosophie; la même notion implique aussi la quête de ce qui est prioritaire, essentiel, la science passe alors au code normatif.

La physiocratie, de fait, prétend découvrir un fonctionnement économique idéaltypique. Si les constats empiriques s'écartent des propositions déduites de la théorie, le démenti n'infirme rien. On en conclura seulement que la société, malade, doit être soignée. Cette opposition entre le normal et le pathologique provient sans doute d'un transfert analogique ; on se souvient que François Quesnay était chirurgien médecin. Mais il entraîne une importante conséquence : l'économie n'est pas réfutable par la statistique, elle traite de ce qui doit être.

Dans ces conditions, on comprend mieux où les physiocrates placent le bon usage de l'Arithmétique politique qu'ils appellent euxmêmes calcul économique. Les quantités qui seront observées pour former des séries statistiques doivent être définies ex ante, conformément à la théorie. Rien de plus légitime, il faut enregistrer par exemple

40. Condorcet, Esquisse d'un tableau historique..., Paris, an III, p. 290-293. Sur la langue universelle, cf. ibid., p. 378 ; sur sa pénétration progressive dans les sciences morales, cf. ibid., p. 251. 
le produit brut au prix du vendeur, les avances annuelles et primitives au prix du consommateur et au taux courant de l'intérêt ${ }^{41}$. Mais les résultats ne contribuent pas à l'édification de la science, ils sont voués à faire éternellement figure d'exceptions aux lois, à dévoiler des écarts. Il apparaît donc une opposition complète entre le point de vue des physiocrates et des arithméticiens, disciples de W. Petty, pour lesquels la science économique repose sur la collecte des données et l'usage de l'induction amplifiante au sens de J. S. Mill.

Dans l'Encyclopédie méthodique, deux textes manifestent emblématiquement ce contraste. Dans la partie Économie politique, Grivel, physiocrate de stricte observance, a rédigé un article «Arithmétique politique " où se trouvent ramassées les critiques de Quesnay. Dans la partie Mathématiques figure un autre article " Arithmétique politique ", rédigé pendant la même année 1784 . Ce dernier assigne à la statistique le rôle que les physiocrates réservent à la théorie :

" On conçoit aisément, écrit-on, que ces découvertes [...] étant acquises par des calculs fondés [...] un ministre habile en tirerait une foule de conséquences pour la perfection de l'agriculture, pour le commerce [...] pour les colonies, pour les cours et l'emploi de l'argent. »

Or ce deuxième texte appartient à Condorcet. Au moment où l'académicien persiste plusieurs années encore à se référer aux principaux théorèmes de la physiocratie, il en a donc abandonné l'épistémologie normative. Depuis combien de temps déjà ? La réponse restera évasive pour l'instant.

Au préalable, il est utile de parcourir le versant philosophique des sciences morales et politiques physiocrates qui fonde en raison cette normativité. Si le Tableau économique résiste à toute réfutation, on l'a dit, c'est qu'il est fondé sur une évidence " mathématique ". Le Mercier de La Rivière introduit ce commentaire : « Elle est donc une autorité despotique, cette force irrésistible de l'évidence " et plus loin : « Euclide est un véritable despote [...] les vérités géométriques qu’il nous a transmises sont des lois véritablement despotiques ${ }^{42}$. François Quesnay a revu ce texte ${ }^{43}$. Le recours à un tel critère est pertinent

41. Pour s'en convaincre, relire les « Questions intéressantes sur la population, l'agriculture et le commerce proposées aux académies et aux autres sociétés savantes des provinces " en 1758, cf. François Quesnay et la physiocratie, Paris, I.N.E.D., 1958, 2 vols, t. II, p. 619-666.

42. P. P. F. J. H. LE MERCIER DE LA RIVIËR, L'Ordre naturel et essentiel des sociétés politiques, Londres, Paris, 1767, 2 vols, t. I, p. 280, 311.

43. L'article « Évidence » de Quesnay figure dans François Quesnay et la physiocratie, op. cit. supra n. 41, t. II. Ce texte est de janvier 1756. Avec « Fermiers » et "Grains », il constitue le coup d'envoi de la doctrine. 
en économie comme dans les sciences de la nature parce qu'il s'adosse à l'hypothèse de l'unité du réel et des procédés de la raison. L'individu en société appartient au monde physique, comme les corps célestes ; le même déterminisme causal peut s'y déchiffrer.

Dans l'Avertissement du premier numéro des Éphémérides du citoyen en 1769, Dupont de Nemours insiste sur cet axiome, le seul à ses yeux qui puisse fonder une science de l'homme :

"On commence à reconnaître qu'il ne peut rien y avoir d'arbitraire dans les règles qui doivent guider la conduite réciproque des hommes et même celle des sociétés [...] Les moyens qui peuvent assurer notre bonheur et celui de notre espèce renferment un enchaînement de causes et d'effets, nécessairement déterminés par des lois également irrésistibles et invariables qui se fondent sur notre nature et sur celle des autres êtres dont nous sommes environnés ${ }^{44}$.

On entendra donc le "despotisme légal " des physiocrates comme celui qui régente la production et la distribution des richesses au même titre que l'attraction, la chute des corps. En surplomb, le système des lois positives, juridiques ou politiques, est entraîné par ce premier moteur, il en prolonge les effets sous peine d'erreur et de faute :

"Le despotisme naturel de l'évidence amène le despotisme social, l'ordre essentiel de toute société est un ordre évident, et comme l'évidence a toujours la même autorité, il n'est pas possible que l'évidence de cet ordre soit manifeste et publique sans qu'elle gouverne despotiquement ${ }^{45}$.

Ainsi l'économie, domaine où l'activité humaine dépend de la matière, asservit à ses lois tout le champ social et politique. La physiocratie est un économisme, l'intérêt que Marx lui porte dans ses Theorien über den Mehrwert est bien justifié.

C'est à la lumière de cette ambition réductrice que l'échec commun de Turgot et de Condorcet peut être réexaminé. En 1776 le ministre et son entourage ont manqué à rendre "manifeste et publique " leur analyse économique. Condorcet le reconnaîtra dix ans plus tard. Dans l'opinion, remarque-t-il,

"Lui [Turgot] et les hommes qui partageaient ses principes y étaient peints comme des gens occupés de chimères systématiques, voulant gou-

44. Ephémérides du citoyen, 1769, no 1, p. VI.

45. P. P. F. J. H. Le Mercier de LA Riviėre, op. cit. supra n. 42, t. I, p. 280. 
verner du fond de leur cabinet d'après des principes spéculatifs et sacrifiant le peuple à des expériences qu'ils voulaient faire pour prouver la vérité de leurs systèmes ${ }^{46}$.

Autrement dit, la valeur de l'évidence n'est pas évidente dans les sciences économiques et sociales. Tant que l'obscurité subsiste sur la nature de l'homme, ses mobiles et ses pratiques sociales, le déterminisme causal des physiocrates n'est pas plus assuré que n'importe quelle autre interprétation. Et de surcroit, l'économie, comme dernière instance explicative des sociétés, devient problématique. Souvenonsnous de la lettre où Condorcet commente le renvoi de Turgot : " Cet événement a changé pour moi toute la nature [...] je vais me remettre à la géométrie et à la philosophie ", c'est-à-dire à l'épistémologie.

Si la théorie économique ne s'impose pas comme celle de Newton, c'est que le savoir et sa transmission même sont parties prenantes dans l'objet scientifique. Les deux échecs analogues de Michel de l'Hospital et de Turgot ne prouvent pas leur tort mais leur incapacité à munir leur analyse de la force décisive qui l'aurait faite vérité. Dans l'économie de Quesnay, aucun effet d'annonce ne pouvait avoir lieu, la découverte des lois de la gravitation ne modifie pas non plus le mouvement des pommes. Mais, en 1776, Condorcet bute sur l'historicité contraignante de la société. L'académicien pressent dès lors l'interaction de la "physique " économique et de ce domaine que le $\mathrm{xX}^{\mathrm{e}}$ siècle explore au moyen de la théorie de l'information et des anticipations.

En vérité, F. Quesnay et ses disciples n'ont pas douté qu'il leur fallait emporter l'adhésion de souverains circonvenus par les financiers et de peuples ignorants. Ils sont les premiers avocats d'un enseignement économique, ils donnent des leçons d'ailleurs médiocrement suivies, ils publient des manuels ${ }^{47}$. Mais ce professorat demeure l'affaire de quelques-uns : Charles de Butré, l'abbé Choquart, Court de Gébelin. L'instruction est une activité utile, elle n'entre pas à titre théorique dans l'économie politique. Un despote éclairé qui obéit à la nature sera suffisant.

Condorcet s'est engagé peu à peu sur la voie inverse. Au terme du parcours, à la publication de sa Mathématique sociale de 1793, l'activité cognitive est devenue l'objet principal du savoir. La formation et la transmission des choix économiques participent à une science «morale " dont la production et la circulation des richesses ne sont

46. Euvres, t. V, Vie de Turgot, p. 101.

47. Cf. J. HeCHT, art. cit. supra n. 34, p. 5-62. L'auteur examine les tentatives d'enseignement économique au xvilIe siècle. Cf. aussi, G. Weulersse, Le Mouvement physiocratique..., op. cit. supra n. 7, t. I, p. 133 sq. 
plus qu'un élément. Même idée dans les cinq « Mémoires sur l'instruction " publiés en 1791 (Bibliothèque de l'homme public)et dans le Rapport et projet de décret sur l'organisation générale de l'Instruction publique, d'avril $1792^{48}$. Condorcet réserve à l'économie et à l'arithmétique politique un double rôle. Ces disciplines, nécessaires au savant comme au citoyen, constituent les clés de politiques économiques réussies, mais elles sont surtout les matériaux d'une réflexion critique tant qu'elles se gardent de prendre des opinions pour des vérités, qu'elles tendent à dissiper « les convictions intrépides " des ignorants ${ }^{49}$.

Est-il excessif de dire que la mathématique sociale contient les prolégomènes de la théorie économique ?Qu'elle prétend donner l'inventaire de ses conditions de possibilité et de ses limites ? Celles-ci sont à ce point historiques, l'Esquisse... des progrès de l'esprit humain le montre bien, que les propositions de l'économie sont destinées au dépérissement dès le moment où elles sont formulées. Turgot avait pressenti ce retard irrémédiable de la raison lorsqu'il écrivait que l'homme politique est, en somme, obligé de prévoir même le présent. C'est admettre que le déterminisme ou le finalisme ne décrivent les sociétés qu'à titre d'illusion rétrospective. En 1793, Condorcet est persuadé que celles-ci instituent au contraire leurs règles de fonctionnement par l'observation réitérée de leurs propres pratiques ; elles s'expliquent à ellesmêmes, elles se ratifient. Cette intuition figure aussi chez Turgot dès 1750 : « Tous les âges sont enchaînés les uns aux autres par une suite de causes et d'effets qui lient l'état présent du monde à tous ceux qui l'ont précédé " ${ }^{50}$.

La recherche de l'ordre naturel immuable est donc vide de sens, elle se trompe de domaine d'application. De là le faible pouvoir prédictif $\mathrm{du}$ raisonnement causal. Les énoncés performants porteront au contraire sur des séries de mesures aussi nombreuses que possible, l'arithmétique établira "leur probabilité par les événements " pour reprendre le titre de la communication de Laplace à l'Académie en 1774. Résumons l'écart des épistémologies successives de Condorcet : celle des physiocrates dont il serait parti en 1775 et celle du Tableau

48. Euvres, t. VII, p. 171-573, notamment dans le deuxième " Mémoire sur l'instruction ", cf. "Sur la nécessité de l'arithmétique politique », p. 280 sq. : réduire le savoir en table, en tirer les résultats et la science de leurs combinaisons. Sinon, « une grande partie des vérités et des opérations qui influent le plus sur le bonheur des hommes seront pour eux une espèce de mystère et [...] ils seront forcés de choisir entre la défiance stupide de l'ignorance et une confiance aveugle. Ils seront toujours exposés à être trompés, soit qu'ils s'abandonnent à suivre une route qu'ils ne connaissent pas, soit qu'ils refusent de s'y engager " (op. cit., p. 282).

49. CondorCET, «Tableau général de la science... », op. cit. supra n. 20, p. 126.

50. G. SCHELle, op. cit. supra n. 13, « Tableau philosophique des progrès successifs de l'esprit humain $»$, décembre 1750 , p. 215. 
de 1793, que vont partager tant d'Idéologues, de Lacretelle, Volney, l'abbé Grégoire, etc. ${ }^{51}$.

1775

Objet fondamental ..... L'économie

Méthode ........... Analyse conceptuelle

+ déduction

Critère ........... L'évidence

Objectif .......... La vérité

Position dans L'économie, dernière

le savoir ........... instance du social

Philosophie générale ... Le monde est compliqué mais divisible en éléments simples, objets de relations causales
1793

L'homme en société

Collecte de données

+ induction

La probabilité

La pertinence

L'interdépendance de toutes les sciences morales et politiques

Le monde est complexe. Son histoire est imprévisible mais non improbable

Voici un tableau qui durcit sans doute l'incompatibilité des deux démarches. Acceptons-le provisoirement. Deux questions restent alors en suspens ; elles ne peuvent plus être éludées. Quel est le degré d'originalité du second Condorcet? Comment s'articule le passage d'une épistémologie à l'autre, à quelle date ?

L'académicien n'est pas l'inventeur de l'approche à laquelle il s'est rallié finalement. On a constaté qu'il avait pratiqué l'ouvrage de JeanFrançois Melon, Essai politique sur le commerce. Or ce texte de la première moitié du XvIIIe siècle préconise déjà la jonction de l'économie avec le calcul des probabilités.

" Tout est réductible au calcul, écrivait cet ancien secrétaire de John Law, il s'étend jusqu'aux choses purement morales. On peut trouver les plus grandes probabilités selon lesquelles un législateur, un ministre, un particulier se déterminera à rejeter ou à accepter une proposition, une entreprise, etc. "

Et tout naturellement l'arithmétique politique est l'outil principal du gouvernement :

« Il doit déterminer son choix sur la pluralité des possibilités où entrent le calcul des hommes, le nombre des travailleurs, la valeur des travaux, le moyen de les multiplier et de les faire valoir ${ }^{52}$.

51. Nombreuses références dans Georges GUSDORF, Les Sciences humaines et la pensée occidentale. T. VIII : La Conscience révolutionnaire, les Idéologues, Paris, Payot, 1978, section II, chap. "Sciences de l'homme », p. 384-427.

52. J.-F. MELon, Essai politique sur le commerce, éd. revue, Amsterdam, 1754, p. 293. 
On s'approche ici, au plus près, de la contribution de Condorcet dans l'Encyclopédie méthodique. Quant au projet d'institution d'une science de l'homme, c'est celui du Traité de la nature humaine. Condorcet connaît bien les livres de Hume. Il y trouve le programme de l'unification des sciences et la méthode, un empirisme logique dont le premier travail est l'analyse critique de la langue ${ }^{53}$. Par conséquent, l'académicien pouvait se mettre à tout instant au travail dans un cadre préalablement connu et formulé.

Ceci explique que la date à laquelle Condorcet s'est engagé sur cette voie soit beaucoup plus ancienne qu'il n'y paraissait à première vue. Il faut la placer vers 1772 , au moment où il se tourne vers le calcul des probabilités en même temps que Laplace, et bien avant la rédaction de ses premiers textes économiques. C'est en 1772 aussi que se place l'épigraphe du présent article, où Condorcet se défend absolument d'adhérer à l'ordre éternellement harmonieux que les physiocrates postulent aux origines de leur philosophie ; dès cette lettre à Turgot, le mécanisme imparfait de notre monde se décrit dans une langue raturée, approximative, approchée, en un mot probabiliste. On sait encore qu'en 1774 Condorcet demande au ministre d'engager des mathématiciens pour les calculs statistiques empiriques ; en 1776, il est convaincu que cette démarche est la meilleure possible :

"Tous ceux qui ont attaqué la certitude des connaissances humaines ont commis la même faute. Ils ont fort bien établi que nous ne pouvons parvenir [...] dans les sciences morales à cette certitude rigoureuse des propositions de la géométrie et cela n'était pas difficile; mais ils ont voulu en conclure que l'homme n'avait aucune règle sûre pour asseoir son opinion sur ces objets et ils se sont trompés en cela. Car il y a des moyens sûrs de

53. David Hume, Traité de la nature humaine, trad. franç. de A. Leroy, Paris, Aubier, 1946, 2 vols. L'objectif est fixé dès l'introduction. Cf. aussi, Didier Deleule, Hume et la naissance du libéralisme économique, Paris, Aubier-Montaigne, 1979. Maupertuis a signalé à ses contemporains le rôle de J.-F. Melơn dans l'introduction de l'épistémologie anglaise en France, dans les sciences économiques: "On éprouva le sentiment qu'une science nouvelle, encore innommée, presque populaire chez les Anglais, mais peu cultivée chez nous, passait la mer et s'installait en France " (cité par G. WeULERSSE, Le Mouvement physiocratique..., op. cit. supra n. 7, t. J, p. 16). Le pari de l'unité de la science est à la base du projet encyclopédique. On se rappelle ce passage du Discours préliminaire où d'Alembert, maître de Condorcet, avance la fameuse proposition «L'Univers pour qui saurait l'embrasser d'un seul point de vue ne serait, s'il est permis de le dire, qu'un fait unique et une grande vérité ». Espoir dont les économistes n'avaient pas tous perdu la nostalgie au xIXe siècle. Dans son "Esquisse d'une doctrine", in Études d'économie politique appliquée, $2 e$ éd., Lausanne/Paris, Pichon/Durand-Auzias, 1936, p. 493, Léon WALRAS formule ce souhait : « Peut-on espérer qu'un jour, la science pure et la science morale achevées l'une et l'autre ne seront plus qu'une science unique?" 
parvenir à une très grande probabilité dans plusieurs cas, et dans un grand nombre, d'évaluer le degré de cette probabilité ${ }^{54}$.

Conclusion : dès le début de sa vie savante, Condorcet professe en même temps une parfaite orthodoxie physiocratique et une épistémologie qui la dément en tout point. Il ne faut donc plus se demander s'il a évolué, mais s'il se contredit. Comment penser ensemble le théoricien de la connaissance aux convictions invariables et l'économiste aux théories fluctuantes? Dira-t-on que l'urgence des événements l'incite à s'engager dans les débats économiques sans qu'il en ait élaboré tous les principes ? Condorcet aurait été physiocrate ou smithien faute de mieux. Durant le ministère Turgot, le défi consistait à mettre en place une régulation libérale de l'économie (suppression des corvées, des corporations, liberté du commerce) et la réforme des finances publiques avec le consentement d'une pyramide d'assemblées. La physiocratie détenait toutes les clés théoriques de ce programme. De son succès dépendait la transition pacifique de la société vers un régime post-absolutiste. Le départ du contrôleur général signifie l'abandon d'une solution "à l'anglaise». Dix ans plus tard, les mêmes nécessités économiques se transcrivent dans le langage de la rupture politique. Ce n'est donc pas seulement à l'enseignement des sciences mais à la marche de la société entière que peuvent se rapporter les propos de Condorcet au début de la Révolution : "Longtemps j’ai considéré ces vues comme des rêves qui ne devaient se réaliser que dans un avenir indéterminé et pour un monde où je n'existerais plus. Un heureux événement a tout à coup ouvert une carrière immense aux espérances du genre humain " ${ }^{55}$. Le jour est revenu où le savant doit accepter de bon cceur les infortunes théoriques, admettre humblement l'éclectisme, choisir la politique économique contre l'économie politique.

Cette modestie scientifique est sans doute adéquate à la chronologie des œuvres de Condorcet, comme aux coups de vent théoriques qui les traversent. Mais l'interprétation ressemble à ces pelisses trop vagues qui vont à tout le monde. Essayons une dernière fois de la resserrer.

On peut montrer d'abord qu'une grande prudence empirique, contre l'opinion françaíse du xvII siècle, caractérisait bien de longue date l'entourage de Turgot. Le ministre a toujours demandé à ses collaborateurs des analyses partielles, circonstanciées; il les presse

54. QEuvres, t. III, Remarques sur les pensées de Pascal, 1776, p. 641. L'intérêt de l'académicien pour l'application des probabilités à l'économie est devenu progressivement exclusif. En 1785, l'année où paraî́t l'Essai sur l'application de l'analyse..., il écrit au marquis Lucchesini : l'Arithmétique politique «est à présent l'objet presque unique de mes recherches ".

55. Euvres, t. VII, cinquième "Mémoire sur l'instruction relative aux sciences", p. 434 . 
d'abandonner les béquilles de la théorie : « Il faut saisir les anneaux qui sont à notre portée et renoncer le plus souvent à voir leur liaison sans s'obstiner à mettre des liaisons artificielles pour arranger des tableaux qui sont toujours plus ou moins arbitraires ". "Vous mettez dans votre ouvrage bien de l'esprit qui sera en pure perte ", ajoute-t-il à l'intention de Dupont de Nemours ${ }^{56}$. Cette mise en garde de 1773 constitue le point de départ épistémologique de Condorcet. Il s'agit d'accepter l'inachèvement des connaissances, leur pertinence approximative, leurs incompatibilités provisoires, de résister au confort des synthèses prématurées, de s'expliquer à soi-même la relativité historique des sciences. Cet effort de pensée a demandé vingt ans de travail au mathématicien.

Le but est atteint dans le Tableau général qui a pour objet l'application du calcul aux sciences politiques et morales. L'auteur est enfin parvenu à se représenter la société sans omettre aucune des activités des sujets concrets. Celles-ci se développent dans trois espaces interdépendants : l'homme, les hommes, les choses. 1) Sur les objets matériels, l'homme agit par le travail, l'emploi des techniques, la répartition de ressources limitées, c'est le champ de l'économie. 2) Avec les hommes, le citoyen entre en interaction au sein de la famille, de la profession, de la société civile et politique, il participe à l'expression d'une volonté collective : la théorie du suffrage est au cœur des sciences morales. 3) Pour lui-même enfin, l'individu se construit une représentation du monde : c'est par le biais des "motifs de croire" que Condorcet rencontre les sciences cognitives.

Aucune hiérarchie n'est visible entre des niveaux qui communiquent les uns avec les autres et s'appréhendent par la même méthode analytique. Cette épistémologie se refuse à distinguer entre infrastructure et superstructure. Il n'existe donc pas de point de départ privilégié sur le circuit des sciences de l'homme. En se consacrant à la recherche d'une politique socio-économique depuis 1774, Condorcet peut paraître céder aux événements et perdre le self-arbitre du savant, mais il rencontre de fait le problème scientifique essentiel du Tableau: la politique économique mobilise les trois instances en même temps. Elle suppose l'analyse de la richesse des nations, l'étude et la pédagogie des interactions sociales, la représentation cognitive des phénomènes ; elle nourrit et critique un savoir économique transitoire, ouvert, dont l'avenir reste " inassignable ", le mot clé de la dixième époque de l'Esquisse.

56. G. SCHELle, op. cit. supra n. 13, t. III, p. 629, lettre du 12 nov. 1773. L'intendant de Limoges vient de recevoir la Table des principes de l'économie politique de Dupont de Nemours. 
Ainsi "la mathématique sociale doit se borner à une exposition générale des méthodes". Elle s'affiche comme critique de la raison économique. C'est seulement le mode de production des théories qui fait aux yeux de Condorcet, l'objet d'une science éventuellement cumulative, tandis que leur contenu retourne inlassablement à l'histoire, à la généalogie, à l'herméneutique : le déchiffrement des progrès de l'esprit. C'est l'Esquisse ${ }^{57}$.

S'il n'existe en économie que des phénomènes momentanés, si leur observation ne peut être réitérée "toutes choses égales", si leur appréhension relève du probable plus que du certain, on conçoit l'embarras des spécialistes lorsqu'il s'agit d'assigner à Condorcet une place de théoricien. En replaçant ce savoir dans l'ensemble des sciences sociales, l'académicien s'offrait de toute manière au risque de l'incompréhension. N'est-ce pas infliger à la discipline la pire des mortifications puisqu'elle aspire depuis 1750 à l'autonomie ? On augure mal d'une telle proposition. Circonstance aggravante, Condorcet propose de généraliser le recours au calcul des probabilités. Pour l'élite raréfiée des géomètres à l'Académie des sciences, cette branche des mathématiques vient de conquérir son statut. Mais dans la cité, combien le perçoivent ? Parmi les administrateurs, les commis de l'État, les "philosophes " qui écrivent, les probabilités demeurent l'ingénieux bricolage qui permet de sophistiquer les rentes viagères, les tontines, les assurances et les loteries ; toutes ces institutions d'ailleurs sont nées sans elles, par tâtonnement. On ne pouvait imaginer que Condorcet se heurte à une plus profonde surdité sociologique et culturelle. La mésaventure des probabilités se répétera longtemps encore. Au XIX ${ }^{e}$ siècle, les meilleurs savants, Say, Cournot, Walras, refusent toujours de faire une place à la statistique ${ }^{58}$.

Jean-Claude Perrot,

Université de Paris-I, E.H.E.S.S.

57. Cette interprétation s'éclaire à la lumière d'un contemporain que Condorcet ne semble pas avoir connu, Immanuel Kant. Il s'agit ici également de débarrasser les sciences de la société de leur sommeil dogmatique et de construire les conditions a priori de l'investigation. D'autre part, l'analyse des trois domaines de rencontre de l'homme avec les choses, avec les autres hommes et avec lui-même englobe tout ce qu'on peut dire de la réalité. Elle présente de l'analogie avec la démarche de Hegel à léna, entre 1801 et 1806. Cf. Jean-Marc FERRY, "L'anthropologie philosophique : un héritage des métaphysiques humanistes pour les sciences humaines", Revue de synthèse, 4, oct.-déc. 1986, p. 361-384.

58. Claude Ménard, « Trois formes de résistance aux statistiques, Say, Cournot, Walras ", Pour une histoire de la statistique, nouv. éd., Paris, Economica, 1987, p. 417-429. 